\title{
A few remarks on the generalized Novikov equation
}

\author{
Shouming Zhou ${ }^{1 *}$ and Rong Chen ${ }^{2}$
}

"Correspondence:

zhoushouming76@163.com

${ }^{1}$ College of Mathematics Science,

Chongqing Normal University,

Chongqing, 401331, P.R. China

Full list of author information is

available at the end of the article

\begin{abstract}
This paper deals with the Cauchy problem for a generalized Novikov equation $u_{t}-u_{x x t}+(b+1) u^{2} u_{x}=b u u_{x} u_{x x}+u^{2} u_{x x x}$, where $b$ is a constant. The local well-posedness in the critical Besov space $B_{2,1}^{3 / 2}$ is established. Moreover, a lower bound for the maximal existence time and lower semicontinuity of the existence are derived, the multi-peakon solutions are also obtained. Finally, the persistence properties in weighted spaces for the solution of this equation are considered.
\end{abstract} MSC: $35 \mathrm{G} 25 ; 35 \mathrm{~L} 05 ; 35 \mathrm{Q} 50$

Keywords: persistence properties; local well-posedness; blow-up

\section{Introduction}

The present paper focuses on the Cauchy problem for the following modified Novikov equation:

$$
\begin{cases}u_{t}-u_{x x t}+(b+1) u^{2} u_{x}=b u u_{x} u_{x x}+u^{2} u_{x x x}, & t>0, x \in \mathbb{R} \\ u(x, 0)=u_{0}(x), & x \in \mathbb{R},\end{cases}
$$

where $b, k_{1}$ and $k_{2}$ are arbitrary constants. Our main purpose of this paper is to establish the well-posedness in the critical Besov space $B_{2,1}^{3 / 2}$ and persistence in a weighted Sobolev space.

Note that when we take $b=3$, Eq. (1.1) is the Novikov equation:

$$
u_{t}-u_{x x t}+4 u^{2} u_{x}=3 u u_{x} u_{x x}+u^{2} u_{x x x}, \quad t>0, x \in \mathbb{R},
$$

which was recently discovered by Novikov in a symmetry classification of nonlocal PDEs with quadratic or cubic nonlinearity [1]. The perturbative symmetry approach [2] yields necessary conditions for a PDE to admit infinitely many symmetries. Using this approach, Novikov was able to isolate Eq. (1.2) and find its first few symmetries, and he subsequently found a scalar Lax pair for it, proving that the equation is integrable. By using the prolongation algebra method, Hone and Wang [3] gave a matrix Lax pair and many conserved densities and a bi-Hamiltonian structure of the Novikov equation, and they showed how it was related by a reciprocal transformation to a negative flow in the Sawada-Kotera hierarchy. Then in [4], the authors calculated the explicit formulas for multi-peakon solutions of the Novikov equation.

\section{Springer}

C2013 Zhou and Chen; licensee Springer. This is an Open Access article distributed under the terms of the Creative Commons Attribution License (http://creativecommons.org/licenses/by/2.0), which permits unrestricted use, distribution, and reproduction in any medium, provided the original work is properly cited. 
Recently, by the transport equations theory and the classical Friedrichs regularization method, the authors proved that the Cauchy problem for the Novikov equation is locally well posed in the Besov spaces $B_{p, r}^{s}$ (with $1 \leq p, r \leq+\infty$ and $s>\max \{1+1 / p, 3 / 2\}$ in $[5,6]$, and with the critical index $s=3 / 2, p=2$ in [7]). It was also shown in [7] that the Novikov equation associated with the initial value is locally well posed in the Sobolev space $H^{s}$ with $s>3 / 2$ by using the abstract Kato theorem. Two results about the persistence properties of the strong solution for Eq. (1.2) were established in [7]. A Galerkin-type approximation method was used in Himonas and Holliman's paper [8] to establish the well-posedness of Novikov equation (1.2) in the Sobolev space $H^{s}$ with $s>3 / 2$ on both the line and the circle, and in $[9,10]$ the authors proved that the data-to-solution map is not globally uniformly continuous on $H^{s}$ for $s<3 / 2$, this result supplements Himonas and Holliman's works. Tiglay [11] showed the local well-posedness of the problem in Sobolev spaces and the existence and uniqueness of solutions for all time using orbit invariants. For analytic initial data, the existence and uniqueness of analytic solutions for Eq. (1.2) were also obtained in [11]. Analogous to the Camassa-Holm equation, the Novikov equation possesses a blow-up phenomenon [10,12] and global weak solutions [13, 14].

On the other hand, it is well known that the nonlinearity of the following $b$-equation is quadratic:

$$
u_{t}-u_{x x t}+(b+1) u u_{x}=b u_{x} u_{x x}+u u_{x x x}, \quad t>0, x \in \mathbb{R},
$$

which can be derived as the family of asymptotically equivalent shallow water wave equations that emerges at quadratic order accuracy for any $b \neq-1$ by an appropriate Kodama transformation. For the case $b=-1$, the corresponding Kodama transformation is singular and the asymptotic ordering is violated (see [15-17]). Equation (1.2) belongs to the following family of nonlinear dispersive partial differential equations:

$$
u_{t}-\gamma u_{x x x}-\alpha^{2} u_{x x t}=\left(c_{1} u^{2}+c_{2} u_{x}^{2}+c_{3} u u_{x x}\right)_{x}
$$

where $\gamma, \alpha, c_{1}, c_{2}$ and $c_{3}$ are real constants. By using Painlevé analysis in [18-20], there are only three asymptotically integrable within this family: the KdV equation, the CamassaHolm (Eq. (1.3) with $b=2$ ) equation and the Degasperis-Procesi equation (Eq. (1.3) with $b=3$ ). The solutions of the $b$-equation were studied numerically for various values of $b$ in $[21,22]$, where $b$ was taken as a bifurcation parameter. The necessary conditions for integrability of the $b$-equation were investigated in [2]. The $b$-equation also admits peakon solutions for any $b \in \mathbb{R}$ (see $[19,21,22])$. The well-posedness, blow-up phenomena and global solutions for the $b$-equation were shown in [23-25].

Recently, $\mathrm{Mi}$ and $\mathrm{Mu}$ [26] studied the local well-posedness in the Besov space $B_{p, r}^{s}$ with $1 \leq p, r \leq+\infty$ and $s>\max \{1+1 / p, 3 / 2\}$. It is well known that $B_{2,2}^{s}(\mathbb{R})=H^{s}$ and for any $s^{\prime}<3 / 2<s: H^{s} \hookrightarrow B_{2,1}^{\frac{3}{2}} \hookrightarrow H^{\frac{3}{2}} \hookrightarrow B_{2, \infty}^{\frac{3}{2}} \hookrightarrow H^{s^{\prime}}$, which shows that $H^{s}$ and $B_{2,2}^{s}$ are quite close, so here we first establish the local well-posedness in the critical Besov space $B_{2,1}^{3 / 2}$.

Theorem 1.1 Assume that the initial data $u_{0}(x) \in B_{2,1}^{\frac{3}{2}}$. Then there exist a unique solution $u(x, t)$ and a maximal time $T=T\left(u_{0}\right)>0$ to the Cauchy problem (1.1) such that

$$
u=u\left(\cdot, u_{0}\right) \in C\left([0, T] ; B_{2,1}^{\frac{3}{2}}\right) \cap C^{1}\left([0, T] ; B_{2,1}^{\frac{1}{2}}\right) .
$$


Moreover, the solution depends continuously on the initial data, i.e., the mapping

$$
u_{0} \mapsto u\left(\cdot, u_{0}\right): B_{2,1}^{\frac{3}{2}} \mapsto C\left([0, T] ; B_{2,1}^{\frac{3}{2}}\right) \cap C^{1}\left([0, T] ; B_{2,1}^{\frac{1}{2}}\right)
$$

is continuous.

Remark 1.1 Following the proof of Theorem 5.1 in [5] and Theorem 1.3 in [10], one can easily get that Eq. (1.1) is not locally well posed in $B_{2, \infty}^{\frac{3}{2}}$ in the following sense: There exists a global solution $u(t, x) \in L^{\infty}\left(\mathbb{R}^{+} ; B_{2, \infty}^{\frac{3}{2}}\right)$ to Eq. (1.1) such that for any $T>0$ and $\epsilon>0$, there exists a solution $v(t, x) \in L^{\infty}\left(\mathbb{R}^{+} ; B_{2, \infty}^{\frac{3}{2}}\right)$ to Eq. (1.1) with

$$
\|u(0)-v(0)\|_{B_{2, \infty}^{\frac{3}{2}}} \leq \epsilon \text { but }\|u(t)-v(t)\|_{L^{\infty}\left(\mathbb{R}^{+} ; B_{2, \infty}^{2}\right.}^{\frac{3}{2}} \leq 1
$$

Remark 1.2 Theorem 1.1 improves the corresponding result in [26]. On the other hand, noting that the counterexample given in [10] cannot be applied to the case in $B_{2, r}^{3 / 2}$ with $1<r<\infty$, the question of local well-posedness of Eq. (1.1) in $H^{3 / 2}$ remains open. Actually, this is still an open problem for the Camassa-Holm and Novikov equations.

Remark 1.3 Since

$$
\|f g\|_{B_{p, \infty}^{-\frac{1}{p}}} \leq C\|f\|_{B_{p, \infty}^{-\frac{1}{p}}}\|g\|_{B_{p, \infty}^{\frac{1}{p}} \cap L^{\infty}} \quad \text { and }\|f\|_{B_{p, 1}^{\frac{1}{p}}} \leq C\|f\|_{B_{p, \infty}^{\frac{1}{p}}} \log \left(e+\frac{\|f\|_{B_{p, \infty}^{1+\frac{1}{p}}}}{\|f\|_{B_{p, \infty}^{\frac{1}{p}}}}\right)
$$

holds for $1 \leq p \leq \infty$ (see [27]), Theorem 1.1 holds true in the case of $B_{p, 1}^{1+1 / p}$ with $1 \leq p<\infty$. Besides, using similar arguments in [28], Theorem 1.1 can also hold true in the case of $B_{p, r}^{s}$ with $s>\max (1+1 / p, 3 / 2)$. Furthermore, the existence of solutions to Eq. (1.1) holds as the initial data belong to $B_{p, r}^{s} \cap \operatorname{Lip}$ with $s>1$, which covers the corresponding result in [26].

Theorem 1.2 Let $u_{0} \in B_{p, r}^{s}$ with $1 \leq p, r \leq \infty$ and $s>\max (3 / 2,1+1 / p)$, then there exists $a$ lifespan $T_{u_{0}}^{*}>0$ such that

$$
T_{u_{0}}^{*}<\infty \quad \Longrightarrow \quad \int_{0}^{T_{u_{0}}^{*}}\left\|u \partial_{x} u(\tau)\right\|_{L^{\infty}} d \tau=\infty
$$

We now get a lower bound depending only on $\left\|u_{0}\right\|_{L i p}$ for the maximal existence time.

Theorem 1.3 Assume that $u_{0} \in \operatorname{Lip} \cap B_{p, r}^{s}, s>\max \{3 / 2,1+1 / p\}$. Let $T^{*}$ be the maximal existence time of the solution $u$ to Eq. (1.1) with the initial data $u_{0}$. Then $T^{*}$ satisfies

$$
T^{*} \geq \frac{1}{a\left(2\left\|u_{0}\right\|_{L^{\infty}}+\left\|u_{0, x}\right\|_{L^{\infty}}\right)^{2}}
$$

with $a=4 \max \{2 / 3,|b-2|, 2|b| / 3,|6-b| / 3\}$.

Next, we shall derive lower semicontinuity of the existence time, provided the initial data is smooth enough. 
Theorem 1.4 Let $v_{0} \in B_{p, r}^{s}, s>\max \{3 / 2,1+1 / p\}$ and $u_{0} \in B_{p, r}^{s+1}$. Assume that $u$, $v$ are two solutions to Eq. (1.1) with the initial data $u_{0}, v_{0}$. Let $T_{u_{0}}, T_{v_{0}}$ be the maximal existence time of the solution $u, v$. If there exist $T<T_{u_{0}}$ and a constant $C$ such that

$$
\left\|u_{0}-v_{0}\right\|_{B_{p, r}^{s}}<\frac{1}{2 C \int_{0}^{T}\left(\|u(\tau)\|_{B_{p, r}^{s+1}}+\left\|u_{0}-v_{0}\right\|_{B_{p, r}^{s}}\right) \exp \left\{2 C \int_{0}^{\tau}\left\|u\left(\tau^{\prime}\right)\right\|_{B_{p, r}^{s+1}}^{2} d \tau^{\prime}\right\} d \tau},
$$

then Eq. (1.1) has a unique solution $v \in E_{p, r}^{s}(T)$.

In [26], the authors consider the single peakon taking the form $u(t, x)=\sqrt{c} e^{-\left|x-c t-x_{0}\right|}$, $c>0$. Moreover, this peakon solitary is a global weak solution to Eq. (1.1). Next, we discuss the existence of multi-peakon solutions to Eq. (1.1).

Theorem 1.5 Equation (1.1) has peakon solutions of the form:

$$
u(t, x)=\sum_{i=1}^{N} p_{i}(t) e^{-\left|x-q_{i}(t)\right|}
$$

whose positions $q_{t}(t)$ and amplitudes $p_{j}(t)$ are according to the dynamical system

$$
\begin{aligned}
& p_{j}^{\prime}=\left(\sum_{i=1}^{N} p_{i} e^{-\left|q_{j}-q_{i}(t)\right|}\right)^{2}, \\
& q_{j}^{\prime}=(b-2) q_{j}\left(\sum_{i=1}^{N} p_{i} e^{-\left|q_{j}-q_{i}\right|}\right)\left(\sum_{i=1}^{N} p_{i} \operatorname{sgn}\left(q_{j}-q_{i}\right) e^{-\left|q_{j}-q_{i}\right|}\right) .
\end{aligned}
$$

In $[7,29-33]$, the spacial decay rates for the strong solutions to the Camassa-Holm equation, the $b$-equation, and the Novikov equation were established provided that the corresponding initial data decay at infinity. This kind of property is the so-called persistence property. Following the main idea of [33], we also prove the persistence properties in weighted spaces for the solution of Eq. (1.1). However, the hard question is that there are cubic nonlinearities in (1.1) which make the proof very difficult. First, we give the following definition of an admissible weight function.

Definition 1.1 An admissible weight function for Eq. (1.1) is a locally absolutely continuous function $\phi: \mathbb{R} \rightarrow \mathbb{R}$ such that, for some $A>0$ and a.e. $x \in \mathbb{R},\left|\phi^{\prime}(x)\right| \leq A|\phi(x)|$, and that is $v$-moderate for some sub-multiplicative weight function $v$ satisfying $\inf _{\mathbb{R}} v>0$ and

$$
\int_{\mathbb{R}} \frac{v(x)}{e^{|x|}} d x<\infty
$$

We recall that a locally absolutely continuous function is a.e. differentiable in $\mathbb{R}$. Moreover, its a.e. derivative belongs to $L_{\mathrm{loc}}^{1}$ and agrees with its distributional derivative. We can now state our main result on admissible weights.

Theorem 1.6 Let $T>0, s>3 / 2$, and $2 \leq p<\infty$. Let also $u \in C\left([0, T], H^{s}(\mathbb{R})\right)$ be a strong solution of the Cauchy problem for Eq. (1.1) such that $\left.u\right|_{t=0}=u_{0}$ satisfies

$$
u_{0} \phi \in L^{p}(\mathbb{R}) \text { and }\left(\partial_{x} u_{0}\right) \phi \in L^{p}(\mathbb{R})
$$


where $\phi$ is an admissible weight function for Eq. (1.1). Then, for all $t \in[0, T]$, we have the estimate

$$
\|u(t) \phi\|_{p}+\left\|\partial_{x} u(t) \phi\right\|_{p} \geq\left(\left\|u_{0} \phi\right\|_{p}+\left\|\partial_{x} u_{0} \phi\right\|_{p}\right) e^{C M t}
$$

for some constant $C>0$ depending only on $v, \phi$ (through the constants $A, C_{0}, \inf _{\mathbb{R}} v$, and $\left.\int_{\mathbb{R}} \frac{v(x)}{e^{|x|}} d x<\infty\right)$, and

$$
M \equiv \sup _{t \in[0, T]}\left(\|u(t)\|_{\infty}+\left\|\partial_{x} u(t)\right\|_{\infty}\right)<\infty
$$

Remark 1.4 The basic example of the application of Theorem 1.6 is obtained by taking the standard weights $\phi=\phi_{a, b, c, d}(x)=e^{a|x|^{b}}(1+|x|)^{c} \log (e+|x|)^{d}$ with the following conditions:

$$
a \geq 0, \quad c, d \in \mathbb{R}, \quad 0 \leq b \leq 1, \quad a b<1 .
$$

(For $a<0$, one has $\phi(x) \rightarrow 0$ as $|x| \rightarrow \infty$ : the conclusion of the theorem remains true but it is not interesting in this case.) The restriction $a b<1$ guarantees the validity of condition (1.6) for a multiplicative function $v(x) \geq 1$.

The limit case $a=b=1$ is not covered by Theorem 1.1. The result holds true, however, for the weight $\phi=\phi_{1,1, c, d}$ with $c<0, d \in \mathbb{R}$, and $\frac{1}{|c|}<p \leq \infty$, or, more generally, when $(1+|\cdot|)^{c} \log (e+|\cdot|)^{d} \in L^{p}(\mathbb{R})$. See Theorem 1.6 below, which covers the case of such fast growing weights.

Remark 1.5 Let us consider a few particular cases:

(1) Take $\phi=\phi_{0,0, c, 0}$ with $c>0$, and choose $p=\infty$. In this case, Theorem 1.6 states that the condition

$$
\left|u_{0}(x)\right|+\left|\partial_{x} u_{0}(x)\right| \leq C(1+|x|)^{-c}
$$

implies the uniform algebraic decay in $[0, T]$ :

$$
|u(x, t)|+\left|\partial_{x} u(x, t)\right| \leq C(1+|x|)^{-c} .
$$

It is worth pointing out that this is a new result for the Novikov equation.

(2) Choose $\phi=\phi_{a, 1,0,0}$ if $x \geq 0$ and $\phi(x)=1$ if $x \leq 0$ with $0 \leq a \leq 1$. Such weight clearly satisfies the admissibility conditions of Definition 1.1. Applying Theorem 1.6 with $p=\infty$, we conclude that the pointwise decay $O\left(e^{-a x}\right)$ as $x \rightarrow+\infty$ is conserved during the evolution. Similarly, we have persistence of the decay $O\left(e^{-a x}\right)$ as $x \rightarrow-\infty$. Hence, our Theorem 1.6 encompasses also Theorem 4.1 of [7].

Since 'peakon' solution $u(t, x)=\sqrt{c} e^{-|x-c t|}, c>0$ does not satisfy the asymptotic behavior in Theorem 1.2 (see Remark 1.4), the purpose of the next theorem is to establish a variant of this theorem that can be applied to some $v$-moderate weights $\phi$ for which condition (1.6) does not hold. Instead of assuming (1.6), we now put the weaker condition

$$
v e^{-|\cdot|} \in L^{p}(\mathbb{R}) .
$$


It is easily checked that, for any continuous sub-multiplicative weight function $v$, we have

$$
v e^{-|\cdot|} \in L^{1}(\mathbb{R}) \quad \Longrightarrow \quad v e^{-|\cdot|} \in L^{p}(\mathbb{R}), \quad \forall 1 \leq p \leq \infty,
$$

so that condition (1.7) is indeed weaker than condition (1.6) (see [33] for the details).

Theorem 1.7 Let $2 \leq p \leq \infty$ and $\phi$ be a v-moderate weight function as in Definition 1.1 satisfying condition (1.7) instead of (1.6). Let also $\left.u\right|_{t=0}=u_{0}$ satisfy

$$
u_{0} \phi \in L^{p}(\mathbb{R}), \quad u_{0} \phi^{\frac{1}{3}} \in L^{3}(\mathbb{R})
$$

and

$$
\left(\partial_{x} u_{0}\right) \phi \in L^{p}(\mathbb{R}), \quad\left(\partial_{x} u_{0}\right) \phi^{\frac{1}{3}} \in L^{3}(\mathbb{R}) .
$$

Let also $u \in C\left([0, T], H^{s}(\mathbb{R})\right), s>3 / 2$ be the strong solution of the Cauchy problem for Eq. (1.1), emanating from $u_{0}$. Then

$$
\sup _{t \in[0, T]}\left(\|u(t) \phi\|_{L^{p}}+\left\|\partial_{x} u(t) \phi\right\|_{L^{p}}\right)<\infty
$$

and

$$
\sup _{t \in[0, T]}\left(\left\|u(t) \phi^{\frac{1}{3}}\right\|_{L^{3}}+\left\|\partial_{x} u(t) \phi^{\frac{1}{3}}\right\|_{L^{3}}\right)<\infty
$$

Remark 1.6 Like Remark 1.5, Theorem 1.7 not only recovers Theorem 4.2 in [7], but also gives a new asymptotic behavior of solutions to Eq. (1.1).

The plan of this paper is organized as follows. In the next section, the local wellposedness in the critical Besov space $B_{2,1}^{3 / 2}$ is considered and Theorem 1.1 is proved. The blow-up criteria and multi-peakon solutions are obtained in Section 3 and Theorems 1.21.5 are proved. In the last section, the persistence properties in weighted spaces for the solution of Eq. (1.1) are considered, and Theorems 1.6-1.7 are proved.

\section{Local well-posedness in critical Besov spaces}

In this section, we shall establish the local well-posedness of Eq. (1.1) in critical Besov spaces. More precisely, we give the proof of Theorem 1.1. First, we rewrite model (1.1) in the following transports equation form:

$$
\left\{\begin{array}{l}
u_{t}+u^{2} u_{x}+\frac{b-2}{2}\left(1-\partial_{x}^{2}\right)^{-1}\left(\partial_{x} u\right)^{3}+\left(1-\partial_{x}^{2}\right)^{-1} \partial_{x}\left(\frac{b}{3} u^{3}+\frac{6-b}{2} u\left(\partial_{x} u\right)^{2}\right)=0 \\
u(x, 0)=u_{0}(x)
\end{array}\right.
$$

We can easily get the following two lemmas.

Lemma 2.1 Let $u_{0}(x) \in B_{2,1}^{\frac{3}{2}}$. Then there exists a time $T>0$ such that the Cauchy problem (1.1) has a solution $u \in C\left([0, T] ; B_{2,1}^{\frac{3}{2}}\right) \cap C^{1}\left([0, T] ; B_{2,1}^{\frac{1}{2}}\right)$. 
Lemma 2.2 Assume that $u_{0}$ (respectively $\left.v_{0}\right) \in B_{2,1}^{\frac{3}{2}}$ such that $u$ (respectively $\left.v\right) \in L^{\infty}([0, T]$; $\left.B_{2,1}^{\frac{3}{2}}\right) \cap C\left([0, T] ; B_{2,1}^{\frac{1}{2}}\right)$ is a solution to the Cauchy problem (1.1) with the initial data $u_{0}$ (respectively $\left.v_{0}\right)$. Let $w=u-v$ and $w_{0}=u_{0}-v_{0}$. Then, for every $t \in[0, T]$,

$$
\|u(t)-v(t)\|_{B_{p, r}^{s-1}} \leq\left\|u_{0}-v_{0}\right\|_{B_{p, r}^{s-1}} \times \exp \left\{C \int_{0}^{T}\left(\|u(\tau)\|_{B_{p, r}^{s}}^{2}+\|v(\tau)\|_{B_{p, r}^{s}}^{2}\right) d \tau\right\} .
$$

Proof of Lemmas 2.1-2.2 The proof is much similar to the case $u_{0} \in B_{p, r}^{s}, s>\max \left\{1+\frac{1}{p}, \frac{3}{2}\right\}$ $($ see $[5,26])$, thus we omit it here.

Lemma 2.3 For any $u_{0} \in B_{2,1}^{\frac{3}{2}}$, there exist a neighborhood $V$ of $u_{0}$ in $B_{2,1}^{\frac{3}{2}}$ and a time $T>0$ such that for any solution of the Cauchy problem (1.1) $v \in V$, the map

$$
\Phi: v_{0} \mapsto v\left(\cdot, v_{0}\right): V \subset B_{2,1}^{\frac{3}{2}} \mapsto C\left([0, T] ; B_{2,1}^{\frac{3}{2}}\right) \cap C^{1}\left([0, T] ; B_{2,1}^{\frac{1}{2}}\right)
$$

is continuous.

Proof Firstly, we prove the continuity of the map $\Phi$ in $C\left([0, T] ; B_{2,1}^{\frac{1}{2}}\right)$. Fix $u_{0} \in B_{2,1}^{\frac{3}{2}}$ and $\delta>0$. Now we claim that there exist $T>0$ and $M>0$ such that for any $v_{0} \in B_{2,1}^{\frac{3}{2}}$ with $\| v_{0}-$ $u_{0} \|_{B_{2,1}^{\frac{3}{2}}} \leq \delta$, the solution $v=\Phi(v)$ of the Cauchy problem (1.1) belongs to $C\left([0, T] ; B_{2,1}^{\frac{3}{2}}\right)$ and satisfies $\|v\|_{L^{\infty}\left([0, T] ; B_{2,1}^{2}\right)} \leq M$. In fact, according to the proof of the local well-posedness, we have that if we fix $T>0$ such that

$$
0<T<\min \left\{\frac{1}{4 C\left\|v_{0}\right\|_{B_{2,1}^{2}}^{2}}, \frac{1}{2 C}\right\},
$$

then

$$
\|v(t)\|_{B_{2,1}^{\frac{3}{2}}} \leq \frac{\left\|v_{0}\right\|_{B_{2,1}^{\frac{3}{2}}}}{\left(1-4 C\left\|v_{0}\right\|_{B_{2,1}^{2}}^{2} t\right)^{\frac{1}{2}}} \quad \text { for all } t \in[0, T] .
$$

As $\left\|v_{0}-u_{0}\right\|_{B_{2,1}^{\frac{3}{2}}} \leq \delta$, then $\left\|v_{0}\right\|_{B_{2,1}^{\frac{3}{2}}} \leq\left\|u_{0}\right\|_{B_{2,1}^{\frac{3}{2}}}+\delta$. Here, one can choose some suitable constant $C$ such that

$$
T=\frac{3}{16 C\left(\left\|u_{0}\right\|_{B_{2,1}^{\frac{3}{2}}}+\delta+1\right)^{2}}<\min \left\{\frac{1}{4 C\left\|v_{0}\right\|_{B_{2,1}^{2}}^{2}}, \frac{1}{2 C}\right\}
$$

and

$$
M=2\left(\left\|u_{0}\right\|_{B_{2,1}^{\frac{3}{2}}}+\delta\right)
$$

Now, combining the above uniform bounds with Lemma 2.2, we get that

$$
\left\|\Phi\left(v_{0}\right)-\Phi\left(u_{0}\right)\right\|_{L^{\infty}\left(0, T ; B_{2,1}^{\frac{3}{2}}\right)} \leq \delta e^{C\left(2 M^{2}+1\right) T} .
$$

Hence $\Phi$ is Holder continuous from $B_{2,1}^{\frac{3}{2}}$ into $C\left([0, T] ; B_{2,1}^{\frac{1}{2}}\right)$. 
Next we prove the continuity of the map $\Phi$ in $C\left([0, T] ; B_{2,1}^{\frac{3}{2}}\right)$. Let $u_{0}^{\infty} \in B_{2,1}^{\frac{3}{2}}$ and $\left(u_{0}^{(n)}\right)_{n \in \mathbb{N}} \rightarrow u_{0}^{\infty}$ in $B_{2,1}^{\frac{3}{2}}$. Let $u^{(n)}$ be the solution of the Cauchy problem (1.1) with the initial data $u_{0}^{(n)}$. From the above argument, we deduce that for any $n \in \mathbb{N}, t \in T$,

$$
\sup _{n \in \mathbb{N}}\left\|u^{(n)}\right\|_{L_{T}^{\infty}\left(B_{2,1}^{2}\right)} \leq M
$$

Note that to prove $u^{(n)} \rightarrow u^{\infty}$ in $C\left([0, T] ; B_{2,1}^{\frac{3}{2}}\right)$ means to prove $u_{x}^{(n)} \rightarrow u_{x}^{\infty}$ in $C\left([0, T] ; B_{2,1}^{\frac{1}{2}}\right)$. Recall that $\left(v^{(n)}\right) \doteq \partial_{x} u^{(n)}$ solves the linear transport equation:

$$
\left\{\begin{array}{l}
\partial_{t}\left(v^{(n)}\right)+\left(u^{(n)}\right)^{2} \partial_{x}\left(v^{(n)}\right)=f^{(n)}, \\
\left.v^{(n)}\right|_{t=0}=\partial_{x}\left(u_{0}^{(n)}\right),
\end{array}\right.
$$

where

$$
\begin{aligned}
f^{(n)}= & 2\left(u^{(n)}\right)\left(\partial_{x} u^{(n)}\right)^{2}-\frac{(b-2)}{2} \partial_{x}\left(1-\partial_{x}^{2}\right)^{-1}\left(\partial_{x} u^{(n)}\right)^{3} \\
& -\left(1-\partial_{x}^{2}\right)^{-1} \partial_{x}^{2}\left(\frac{b}{3}\left(u^{(n)}\right)^{3}+\frac{6-b}{2}\left(u^{(n)}\right)\left(\partial_{x} u^{(n)}\right)^{2}\right) .
\end{aligned}
$$

Thanks to the Kato theory [28], we decompose $v^{(n)}$ into $v^{(n)}=z^{(n)}+w^{(n)}$ with

$$
\left\{\begin{array}{l}
\partial_{t}\left(z^{(n)}\right)+\left(u^{(n)}\right)^{2} \partial_{x}\left(z^{(n)}\right)=f^{(n)}-f^{\infty}, \\
\left.v^{(n)}\right|_{t=0}=\partial_{x}\left(u_{0}^{(n)}\right)-\partial_{x}\left(u_{0}^{\infty}\right)
\end{array}\right.
$$

and

$$
\left\{\begin{array}{l}
\partial_{t}\left(w^{(n)}\right)+\left(w^{(n)}\right)^{2} \partial_{x}\left(w^{(n)}\right)=f^{\infty}, \\
\left.w^{(n)}\right|_{t=0}=\partial_{x}\left(u_{0}^{\infty}\right) .
\end{array}\right.
$$

According to the first step, we have that the sequence $\left(u^{(n)}\right)_{n \in \overline{\mathbb{N}}}(\overline{\mathbb{N}}=\mathbb{N} \cup\{\infty\})$ is uniformly bounded in $C\left([0, T] ; B_{2,1}^{\frac{3}{2}}\right)$ and tends to $\left(u^{\infty}\right)$ in $C\left([0, T] ; B_{2,1}^{\frac{1}{2}}\right)$, thus we can use Proposition 3 in [27], which implies that $w^{(n)}$ tends to $w^{\infty}$ in $C\left([0, T] ; B_{2,1}^{\frac{1}{2}}\right)$, i.e., for any $\varepsilon>0$, $\| w^{(n)}-$ $w^{\infty} \|_{B_{2,1} \frac{1}{2}} \leq \varepsilon$.

On the other hand, applying Lemma 2.3 in [7] and the product law in the Besov spaces to Eq. (2.5), one may get that

$$
\begin{aligned}
\left\|z^{(n)}\right\|_{B_{2,1}^{\frac{1}{2}}} \leq & \exp \left\{C \int_{0}^{t}\left\|\left(u^{(n)}\right)^{2}(\tau)\right\|_{B_{2,1}^{\frac{3}{2}}} d \tau\right\} \\
& \cdot\left(\left\|\partial_{x}\left(u_{0}^{(n)}\right)-\partial_{x}\left(u_{0}^{\infty}\right)\right\|_{B_{2,1}^{\frac{1}{2}}}+\int_{0}^{t}\left\|f^{(n)}-f^{\infty}\right\|_{B_{2,1}^{\frac{1}{2}}} d \tau\right) .
\end{aligned}
$$

Using the properties of Besov spaces exhibited in [27], one easily checks that $\left(f^{(n)}\right)_{n \in \overline{\mathbb{N}}}$ is uniformly bounded in $C\left([0, T] ; B_{1,2}^{\frac{1}{2}}\right)$. Moreover,

$$
\begin{aligned}
\left\|f^{(n)}-f^{\infty}\right\|_{B_{2,1}^{\frac{1}{2}} \leq} \leq & C\left(\left\|u^{(n)}\right\|_{B_{2,1}^{\frac{3}{2}}}^{2}+\left\|u^{(n)}\right\|_{B_{2,1}^{\frac{3}{2}}}\left\|u^{\infty}\right\|_{B_{2,1}^{\frac{3}{2}}}\right)\left\|\partial_{x}\left(u^{(n)}\right)-\partial_{x}\left(u^{\infty}\right)\right\|_{B_{2,1}^{\frac{1}{2}}} \\
& +C\left\|u^{(n)}-u^{\infty}\right\|_{B_{2,1}^{\frac{1}{2}}\left\|u^{\infty}\right\|_{B_{2,1}^{\frac{3}{2}}}^{2}}
\end{aligned}
$$


Hence, combining the convergence of $z^{(n)}$ in $C\left([0, T] ; B_{2,1}^{\frac{1}{2}}\right)$ with estimates (2.4)-(2.7), we deduce that for large enough $n \in \mathbb{N}$,

$$
\begin{aligned}
\left\|\partial_{x}\left(u^{(n)}\right)-\partial_{x}\left(u^{\infty}\right)\right\|_{B_{2,1}^{\frac{1}{2}} \leq} \leq & +C\left(2 M^{2}+1\right) e^{C\left(2 M^{2}+1\right)}\left[\left\|\partial_{x}\left(u_{0}^{(n)}\right)-\partial_{x}\left(u_{0}^{\infty}\right)\right\|_{B_{2,1}^{\frac{1}{2}}}\right. \\
& \left.+\int_{0}^{t}\left\|u^{(n)}-u^{\infty}\right\|_{B_{2,1}^{\frac{1}{2}}} d \tau+\int_{0}^{t}\left\|\partial_{x}\left(u^{(n)}\right)-\partial_{x}\left(u^{\infty}\right)\right\|_{B_{2,1}^{\frac{1}{2}}} d \tau\right] .
\end{aligned}
$$

Thanks to Gronwall's inequality, we have

$$
\left\|\partial_{x}\left(u^{(n)}\right)-\partial_{x}\left(u^{\infty}\right)\right\|_{L^{\infty}\left(0, T ; B_{2,1}^{\frac{1}{2}}\right)} \leq C(M, m, T)\left(\left\|\partial_{x}\left(u_{0}^{(n)}\right)-\partial_{x}\left(u_{0}^{\infty}\right)\right\|_{B_{2,1}^{\frac{1}{2}}}+\varepsilon\right)
$$

for some constant $C$ depending only on $M$ and $b$. We have completed the continuity of the map $\Phi$ in $C\left([0, T] ; B_{2,1}^{\frac{3}{2}}\right)$.

Now, applying $\partial_{t}$ to Eq. (1.1) and by the same argument to the resulting equation in terms of $\partial_{t} u$, we may check the continuity of the map $\Phi$ in $C^{1}\left([0, T] ; B_{2,1}^{\frac{1}{2}}\right)$.

Proof of Theorem 1.1 Combining the result in Lemma 2.1 with that in Lemma 2.2, one gets the existence and uniqueness of the solution of the Cauchy problem (1.1). And Lemma 2.3 shows that the solution of the Cauchy problem (1.1) depends continuously on the initial data. This completes the proof of Theorem 1.1.

\section{Blow-up criterion and multi-peakon solutions}

This section is devoted to the proof of Theorems 1.2-1.5. Theorems 1.2-1.3 are based on the following lemma.

Lemma 3.1 Let $u_{0} \in B_{p, r}^{s}$ with $1 \leq p, r \leq \infty$ and $s>1$. Let $u \in L^{\infty}\left([0, T] ; B_{p, r}^{s}\right)$ solve Eq. (1.1) on $[0, T) \times \mathbb{R}$ with the initial data $u_{0}$. There exist a constant $C_{1}$ depending only on $s$ and $p$ and a universal constant $C_{2}$ such that for all $t \in[0, T)$, we have

$$
\begin{aligned}
& \|u(t)\|_{B_{p, r}^{s}} \leq\left\|u_{0}\right\|_{B_{p, r}^{s}} \exp \left(C_{1} \int_{0}^{t}\|u(\tau)\|_{L i p}^{2} d \tau\right), \\
& \|u(t)\|_{L i p} \leq\left\|u_{0}\right\|_{L i p} \exp \left(C_{2} \int_{0}^{t}\left\|u \partial_{x} u(\tau)\right\|_{L^{\infty}} d \tau\right) .
\end{aligned}
$$

Proof Applying the last of Lemma 2.3 in [6] to the Novikov equation and using the fact that $\left(1-\partial_{x}^{2}\right)^{-1}$ is a multiplier of order -2 yields

$$
\begin{aligned}
\exp \left(-C \int_{0}^{t}\left\|u \partial_{x} u(\tau)\right\|_{L^{\infty}} d \tau\right)\|u(t)\|_{B_{p, r}^{s}} \\
\leq\left\|u_{0}\right\|_{B_{p, r}^{s}}+C \int_{0}^{t} \exp \left(-C \int_{0}^{\tau}\left\|u \partial_{x} u(\tau)\right\|_{L^{\infty}} d \tau^{\prime}\right) \\
\cdot\left(\left\|u^{3}\right\|_{B_{p, r}^{s-1}}+\left\|u u_{x}^{2}\right\|_{B_{p, r}^{s-1}}+\left\|u_{x}^{3}\right\|_{B_{p, r}^{s-2}}\right) d \tau .
\end{aligned}
$$

As $s-1>0$, according to Lemma 2.2(5) in [6], one gets

$$
\left\|u^{3}\right\|_{B_{p, r}^{s-1}}+\left\|u u_{x}^{2}\right\|_{B_{p, r}^{s-1}}+\left\|u_{x}^{3}\right\|_{B_{p, r}^{s-2}} \leq C\|u\|_{L i p}^{2}\|u\|_{B_{p, r}^{s}} .
$$


Therefore

$$
\begin{aligned}
& \exp \left(-C \int_{0}^{t}\left\|u \partial_{x} u(\tau)\right\|_{L^{\infty}} d \tau\right)\|u(t)\|_{B_{p, r}^{s}} \\
& \quad \leq\left\|u_{0}\right\|_{B_{p, r}^{s}}+C \int_{0}^{t} \exp \left(-C \int_{0}^{\tau}\left\|u \partial_{x} u(\tau)\right\|_{L^{\infty}} d \tau^{\prime}\right)\|u\|_{L i p}^{2}\|u\|_{B_{p, r}^{s}} d \tau .
\end{aligned}
$$

Applying Gronwall's lemma completes the proof of (3.1).

By differentiating once Eq. (1.1) with respect to $x$, and applying the $L^{\infty}$ estimate for transport equations, we easily prove that

$$
\begin{aligned}
\exp \left(-C \int_{0}^{t}\left\|u \partial_{x} u(\tau)\right\|_{L^{\infty}} d \tau\right)\|u(t)\|_{L i p} \\
\leq\left\|u_{0}\right\|_{L i p}+C \int_{0}^{t} \exp \left(-C \int_{0}^{\tau}\left\|u \partial_{x} u(\tau)\right\|_{L^{\infty}} d \tau^{\prime}\right) \\
\quad \cdot\left\|\frac{b-2}{2}\left(1-\partial_{x}^{2}\right)^{-1}\left(\partial_{x} u\right)^{3}+\left(1-\partial_{x}^{2}\right)^{-1} \partial_{x}\left(\frac{b}{3} u^{3}+\frac{6-b}{2} u\left(\partial_{x} u\right)^{2}\right)\right\|_{L i p} d \tau .
\end{aligned}
$$

Since $\left(1-\partial_{x}^{2}\right)^{-1} f=\frac{1}{2} e^{-|x|} * f$ and the Young inequality, we get

$$
\left\|\frac{b-2}{2}\left(1-\partial_{x}^{2}\right)^{-1}\left(\partial_{x} u\right)^{3}+\left(1-\partial_{x}^{2}\right)^{-1} \partial_{x}\left(\frac{b}{3} u^{3}+\frac{6-b}{2} u\left(\partial_{x} u\right)^{2}\right)\right\|_{L i p} \leq C^{\prime}\|u\|_{L i p}^{2}\left\|\partial_{x} u\right\|_{L^{\infty}}
$$

for some universal constant $C^{\prime}$. Hence Gronwall's lemma gives inequality (3.2).

Proof of Theorem 1.2 Let $u \in \bigcap_{T<T^{*}} E_{p, r}^{s}(T)$ be such that $\int_{0}^{T^{*}}\left\|u \partial_{x} u(\tau)\right\|_{L^{\infty}} d \tau$ is finite. According to inequality (3.2), $\int_{0}^{T^{*}}\|u(\tau)\|_{L^{\infty}}^{2} d \tau$ is also finite. Hence, (3.1) insures that

$$
\|u(t)\|_{B_{p, r}^{s}} \leq M_{T^{*}}:=\left\|u_{0}\right\|_{B_{p, r}^{s}} \exp \left(C_{1} \int_{0}^{t}\|u(\tau)\|_{L i p}^{2} d \tau\right)<\infty, \quad \forall t \in\left[0, T^{*}\right) .
$$

Let $\epsilon>0$ be such that $2 C^{2} \epsilon M_{T^{*}}<1$, where $C$ stands for the constants used in the proof of Lemma 2.1 in [26]. We then have a solution $\tilde{u}(t) \in E_{p, r}^{s}(\epsilon)$ to Eq. (1.1) with the initial data $u\left(T^{*}-\epsilon / 2\right)$. For the sake of uniqueness, $\tilde{u}(t)=u\left(t+T^{*}-\epsilon / 2\right)$ on $[0, \epsilon / 2)$ so that $\tilde{u}$ extends the solution $u$ beyond $T^{*}$. We conclude that $T^{*}<T_{u_{0}}^{*}$ and Theorem 1.2 is proved.

Proof of Theorem 1.3 Multiplying Eq. (2.1) by $u^{2 n-1}$ with $n \in \mathbb{Z}^{+}$and integrating by parts, we obtain

$$
\int_{\mathbb{R}} u^{2 n-1}\left(u_{t}+u^{2} u_{x}+F\right) d x=0
$$

with $F=\frac{b-2}{2}\left(1-\partial_{x}^{2}\right)^{-1}\left(\partial_{x} u\right)^{3}+\left(1-\partial_{x}^{2}\right)^{-1} \partial_{x}\left(\frac{b}{3} u^{3}+\frac{6-b}{2} u\left(\partial_{x} u\right)^{2}\right)$. Note that the estimates

$$
\int_{\mathbb{R}} u^{2 n-1} u_{t} d x=\frac{1}{2 n} \frac{d}{d t}\|u(x, t)\|_{L^{2 n}}^{2 n}=\|u(x, t)\|_{L^{2 n}}^{2 n-1} \frac{d}{d t}\|u(x, t)\|_{L^{2 n}}
$$

and

$$
\left|\int_{\mathbb{R}} u^{2 n-1}\left(u^{2} u_{x}\right) d x\right| \leq\left\|u u_{x}(x, t)\right\|_{L^{\infty}}\|u(x, t)\|_{L^{2 n}}^{2 n}
$$


are true. Moreover, using Hölder's inequality

$$
\left|\int_{\mathbb{R}} u^{2 n-1} F d x\right| \leq\|u(x, t)\|_{L^{2 n}}^{2 n-1}\|F\|_{L^{2 n}}
$$

from (2.2) we can obtain

$$
\frac{d}{d t}\|u(x, t)\|_{L^{2 p}} \leq\left\|u u_{x}(x, t)\right\|_{L^{\infty}}\|u(x, t)\|_{L^{2 p}}+\|F\|_{L^{2 p}} .
$$

Since $\|f\|_{L^{p}} \rightarrow\|f\|_{L^{\infty}}$ as $p \rightarrow \infty$ for any $f \in L^{\infty} \cap L^{2}$ and the operator $\left(1-\partial_{x}^{2}\right)^{-1}=\frac{1}{2} e^{-|x|}$, from the above inequality we deduce that

$$
\begin{aligned}
\frac{d}{d t}\|u(x, t)\|_{L^{\infty}} \leq & \left\|u_{x}\right\|_{L^{\infty}}\|u\|_{L^{\infty}}^{2}+\frac{|b-2|}{2}\left\|u_{x}(\tau)\right\|_{L^{\infty}}^{3} \\
& +\frac{|b|}{3}\|u(\tau)\|_{L^{\infty}}^{3}+\frac{|6-b|}{2}\|u(\tau)\|_{L^{\infty}}\left\|u_{x}(\tau)\right\|_{L^{\infty}}^{2} .
\end{aligned}
$$

Next, we give estimates on $\left\|u_{x}(x, t)\right\|_{L^{\infty}}$. Differentiating (2.1) with respect to $x$-variable produces the equation

$$
u_{x t}+u^{2} u_{x x}+2 u u_{x}^{2}+\partial_{x} F=0
$$

Similar to the estimate of (3.4), we deduce that

$$
\begin{aligned}
\frac{d}{d t}\left\|u_{x}(t)\right\|_{L^{\infty}} \leq & 2\left\|u_{x}\right\|_{L^{\infty}}\|u\|_{L^{\infty}}^{2}+\frac{|b-2|}{2}\left\|u_{x}(\tau)\right\|_{L^{\infty}}^{3} \\
& +\frac{2|b|}{3}\|u(\tau)\|_{L^{\infty}}^{3}+|6-b|\|u(\tau)\|_{L^{\infty}}\left\|u_{x}(\tau)\right\|_{L^{\infty}}^{2} .
\end{aligned}
$$

Choose

$$
H(t):=2\|u(t)\|_{L^{\infty}}+\left\|u_{x}(t)\right\|_{L^{\infty}} .
$$

Combining (3.4) with (3.5), we get

$$
\frac{d}{d t} H(t) \leq \frac{a}{2} H^{3}(t)
$$

with $a=4 \max \{2 / 3,|b-2|, 2|b| / 3,|6-b| / 3\}$. Define $T:=\frac{1}{a\left(2\left\|u_{0}\right\|_{L} \infty+\left\|u_{0, x}\right\|_{L} \infty\right)^{2}}$. By (3.6), then for all $t<\min \left\{T, T^{*}\right\}$, one can easily get

$$
H(t) \leq \frac{H(0)}{\sqrt{1-a H^{2}(0) t}} .
$$

Theorem 1.2 yields that $T^{*} \geq T$. This completes the proof of Theorem 1.3.

Proof of Theorem 1.4 Let $w=u-v$. In view of Eq. (2.1), one can get

$$
w_{t}+(u+w)^{2} w_{x}=-(2 u-w) w u_{x}+f+g
$$


with

$$
\begin{aligned}
& f=\left(1-\partial_{x}^{2}\right)^{-1} \partial_{x}\left[\frac{b}{3}\left(u^{3}-v^{3}\right)+\frac{6-b}{2}\left(u\left(\partial_{x} u\right)^{2}-v\left(\partial_{x} v\right)^{2}\right)\right], \\
& g=\frac{b-2}{2}\left(1-\partial_{x}^{2}\right)^{-1}\left[\left(\partial_{x} u\right)^{3}-\left(\partial_{x} v\right)^{3}\right] .
\end{aligned}
$$

Using standard energy arguments and integration by parts, we end up with

$$
\begin{aligned}
\|w(t)\|_{B_{p, r}^{s}} \leq & \left\|w_{0}\right\|_{B_{p, r}^{s}}+c \int_{0}^{t}\left\|(u+w)^{2}(\tau)\right\|_{B_{p, r}^{s}}\|w(\tau)\|_{B_{p, r}^{s}} d \tau \\
& +\int_{0}^{t}\left(\left\|(2 u-w) w u_{x}(\tau)\right\|_{B_{p, r}^{s}}+\|(f+g)(\tau)\|_{B_{p, r}^{s}}\right) d \tau .
\end{aligned}
$$

Note that

$$
\begin{aligned}
& \left\|(u+w)^{2}\right\|_{B_{p, r}^{s}} \leq c\left(\|u\|_{B_{p, r}^{s+1}}^{2}+\|u\|_{B_{p, r}^{s+1}}\|w\|_{B_{p, r}^{s}}+\|w\|_{B_{p, r}^{s}}^{2}\right), \\
& \left\|(2 u-w) w u_{x}\right\|_{B_{p, r}^{s}} \leq c\left(\|u\|_{B_{p, r}^{s+1}}^{2}+\|u\|_{B_{p, r}^{s+r}}\|w\|_{B_{p, r}^{s}}\right)\|w\|_{B_{p, r}^{s}}
\end{aligned}
$$

and

$$
\begin{aligned}
\|f+g\|_{B_{p, r}^{s}} & \leq\|f\|_{B_{p, r}^{s}}+\|g\|_{B_{p, r}^{s}} \\
& \leq c\left(\|u\|_{B_{p, r}^{s+1}}^{2}+\|u\|_{B_{p, r}^{s, 1}}\|w\|_{B_{p, r}^{s}}+\|w\|_{B_{p, r}^{s}}^{2}\right)\|w\|_{B_{p, r}^{s}} .
\end{aligned}
$$

Using the above inequalities, and applying Gronwall's inequality to (3.9), one can easily get that

$$
\begin{aligned}
& \|w(t)\|_{B_{p, r}^{s}} \\
& \quad \leq\left\|w_{0}\right\|_{B_{p, r}^{s}} \exp \left\{c \int_{0}^{t}\left(\|u(\tau)\|_{B_{p, r}^{s+r}}^{2}+\|u(\tau)\|_{B_{p, r}^{s+r}}\|w(\tau)\|_{B_{p, r}^{s}}+\|w(\tau)\|_{B_{p, r}^{s}}^{2}\right) d \tau\right\} .
\end{aligned}
$$

Let

$$
\begin{aligned}
& P(t)=\exp \left\{-2 c \int_{0}^{t}\left(\|u(\tau)\|_{B_{p, r}^{s+1}}\|w(\tau)\|_{B_{p, r}^{s}}+\|w(\tau)\|_{B_{p, r}^{s}}^{2}\right) d \tau\right\}, \\
& Q(t)=c\left(\|u(\tau)\|_{B_{p, r}^{s+r}}\left\|w_{0}\right\|_{B_{p, r}^{s}}+\left\|w_{0}\right\|_{B_{p, r}^{s}}^{2}\right) \exp \left\{2 c \int_{0}^{t}\|u(\tau)\|_{P_{p, r}^{s+1}}^{2} d \tau\right\} .
\end{aligned}
$$

According to (3.10), we obtain

$$
\frac{d P(t)}{d t} \geq 2 Q(t)
$$

Integrating (3.11) on $[0, t]$ with $t<T$, by virtue of (3.10), we get

$$
\|w(t)\|_{B_{p, r}^{s}}^{2} \leq \frac{\left\|w_{0}\right\|_{B_{p, r}^{s}}^{2} \exp \left\{2 c \int_{0}^{t}\|u(\tau)\|_{B_{p, r}^{s+r}}^{2} d \tau\right\}}{1-2 c \int_{0}^{t}\left(\|u(\tau)\|_{B_{p, r}^{s+r}}\left\|w_{0}\right\|_{B_{p, r}^{s}}+\left\|w_{0}\right\|_{B_{p, r}^{s}}^{2}\right) \exp \left\{2 c \int_{0}^{\tau}\left\|u\left(\tau^{\prime}\right)\right\|_{B_{p, r}^{s+1}}^{2} d \tau^{\prime}\right\} d \tau} .
$$


If $T_{v_{0}} \geq T$, for all $t \leq T_{v_{0}}$, the above inequality implies that

$$
\begin{aligned}
\|w(t)\|_{B_{p, r}^{s}}^{2} & \leq \frac{\left\|w_{0}\right\|_{B_{p, r}^{s}}^{2} \exp \left\{2 c \int_{0}^{T_{v_{0}}}\|u(\tau)\|_{B_{p, r}^{s+1}}^{2} d \tau\right\}}{1-2 c \int_{0}^{T_{\nu_{0}}}\left(\|u(\tau)\|_{B_{p, r}^{s+1}}\left\|w_{0}\right\|_{B_{p, r}^{s}}+\left\|w_{0}\right\|_{B_{p, r}^{s}}^{2}\right) \exp \left\{2 c \int_{0}^{\tau}\left\|u\left(\tau^{\prime}\right)\right\|_{B_{p, r}^{s+1}}^{2} d \tau^{\prime}\right\} d \tau} \\
& \leq \infty
\end{aligned}
$$

Therefore, $\|w(t)\|_{B_{p, r}^{s}}$ is uniformly bounded in $\left[0, T_{v_{0}}\right]$. In view of Theorem 1.2 , the solution can be extended beyond $T_{v_{0}}$. This is in conflict with the definition of $T_{v_{0}}$.

Remark 3.1 If $r=1, s=1+1 / p$, in view of $B_{p, 1}^{1 / p}$ being an algebra, we have (3.10). Thus we also deduce the result of Theorem 1.4.

Proof of Theorem 1.5 We now derive the multi-peakon solutions of Eq. (1.1). Assume that Eq. (1.1) has an $N$-peakon solution of the form (1.4). It follows from the definition of a weak solution that for any $\psi(t, x) \in C_{c}^{\infty}([0, \infty) \times \mathbb{R})$, the solution (1.1) satisfies

$$
\begin{aligned}
& \int_{0}^{\infty} \int_{\mathbb{R}}\left[u_{t}+u^{2} u_{x}+\frac{(b-2)}{2}\left(1-\partial_{x}^{2}\right)^{-1}\left(\partial_{x} u\right)^{3}\right. \\
& \left.+\left(1-\partial_{x}^{2}\right)^{-1} \partial_{x}\left(\frac{b}{3} u^{3}+\frac{6-b}{2} u\left(\partial_{x} u\right)^{2}\right)\right] \varphi(x) d x d t=0,
\end{aligned}
$$

which is equivalent to the following equation:

$$
\begin{gathered}
\int_{0}^{\infty} \int_{\mathbb{R}}\left[u_{t}\left(\phi-\phi_{x x}\right)+\frac{1}{3} u^{3} \phi_{x x x}+\frac{(b-2)}{2}\left(\partial_{x} u\right)^{3} \phi\right. \\
\left.-\phi_{x}\left(\frac{b+1}{3} u^{3}+\frac{6-b}{2} u\left(\partial_{x} u\right)^{2}\right)\right] d x d t=0,
\end{gathered}
$$

where $\varphi=\phi-\phi_{x x}, \phi(t, x) \in C_{c}^{\infty}([0, \infty) \times \mathbb{R})$.

A straightforward computation gives

$$
\begin{aligned}
\int_{0}^{\infty} \int_{\mathbb{R}} u_{t}\left(\phi-\phi_{x x}\right) d x d t= & \sum_{i=1}^{N} \int_{0}^{\infty} \int_{-\infty}^{q_{j}(t)}\left(p_{j}^{\prime}-p_{j} q_{j}^{\prime}\right) e^{x-q_{j}}\left(\phi-\phi_{x x}\right) d x d t \\
& +\sum_{i=1}^{N} \int_{0}^{\infty} \int_{q_{j}(t)}^{\infty}\left(p_{j}^{\prime}+p_{j} q_{j}^{\prime}\right) e^{-\left(x-q_{j}\right)}\left(\phi-\phi_{x x}\right) d x d t \\
= & 2 \int_{0}^{\infty} \sum_{i=1}^{N}\left(p_{j}^{\prime} \phi\left(q_{j}\right)+p_{j} q_{j}^{\prime} \phi_{x}\left(q_{j}\right)\right) d t
\end{aligned}
$$

and

$$
\begin{aligned}
\frac{1}{3} \int_{\mathbb{R}} u^{3} \phi_{x x x} d x & =-\left(\int_{-\infty}^{q_{1}}+\sum_{j=1}^{N-1} \int_{q_{j}}^{q_{j+1}}+\int_{q_{N}}^{\infty}\right) u^{2} u_{x} \phi_{x x} d x \\
& =-u^{2} u_{x} \phi_{x}\left(\left.\right|_{-\infty} ^{q_{1}}+\left.\sum_{j=1}^{N-1}\right|_{q_{j}} ^{q_{j+1}}+\left.\right|_{q_{N}} ^{\infty}\right)+\int_{\mathbb{R}}\left(2 u u_{x}^{2}+u^{2} u_{x x}\right) \phi_{x} d x
\end{aligned}
$$




$$
\begin{aligned}
= & {\left[-u^{2} u_{x} \phi_{x}+\left(2 u u_{x}^{2}+u^{3}\right) \phi\right]\left(\left.\right|_{-\infty} ^{q_{1}}+\left.\sum_{j=1}^{N-1}\right|_{q_{j}} ^{q_{j+1}}+\left.\right|_{q_{N}} ^{\infty}\right) } \\
& -\int_{\mathbb{R}}\left(2 u_{x}^{3}+4 u^{4} u_{x}+4 u^{2} u_{x}\right) \phi d x
\end{aligned}
$$

and

$$
\begin{aligned}
- & \int_{\mathbb{R}}\left(\frac{b+1}{3} u^{3}+\frac{6-b}{2} u\left(\partial_{x} u\right)^{2}\right) \phi_{x} d x \\
= & -\left[\left(\frac{b+1}{3} u^{3}+\frac{6-b}{2} u u_{x}^{2}\right) \phi\right]\left(\left.\right|_{-\infty} ^{q_{1}}+\left.\sum_{j=1}^{N-1}\right|_{q_{j}} ^{q_{j+1}}+\left.\right|_{q_{N}} ^{\infty}\right) \\
& +\int_{\mathbb{R}}\left((b+1) u^{2} u_{x}+(6-b) u^{2} u_{x}+\frac{6-b}{2} u_{x}^{3}\right) d x .
\end{aligned}
$$

Thus, combining (3.15) with (3.16), we get

$$
\begin{aligned}
\int_{\mathbb{R}} & {\left[\frac{1}{3} u^{3} \phi_{x x x}+\frac{b-2}{2}\left(\partial_{x} u\right)^{3} \phi-\phi_{x}\left(\frac{b+1}{3} u^{3}+\frac{6-b}{2} u\left(\partial_{x} u\right)^{2}\right)\right] d x } \\
= & \left(-u^{2} u_{x} \phi_{x}+\frac{b-2}{2} u u_{x}^{2} \phi\right)\left(\left.\right|_{-\infty} ^{q_{1}}+\left.\sum_{j=1}^{N-1}\right|_{q_{j}} ^{q_{j+1}}+\left.\right|_{q_{N}} ^{\infty}\right) \\
= & -2 \sum_{j=1}^{N}\left[p_{j}\left(\sum_{i=1}^{N} p_{i} e^{-\left|q_{j}-q_{i}\right|}\right)^{2} \phi_{x}\left(q_{j}\right)\right] \\
& -2(b-2) \sum_{j=1}^{N}\left[p_{j}\left(\sum_{i=1}^{N} p_{i} e^{-\left|q_{j}-q_{i}\right|}\right)\left(\sum_{i=1}^{N} p_{i} \operatorname{sgn}\left(q_{j}-q_{i}\right) e^{-\left|q_{j}-q_{i}\right|}\right) \phi\left(q_{j}\right)\right] .
\end{aligned}
$$

Substituting (3.14), (3.17) into (3.13), we obtain the following system:

$$
\begin{aligned}
& p_{j}^{\prime}=\left(\sum_{i=1}^{N} p_{i} e^{-\left|q_{j}-q_{i}(t)\right|}\right)^{2}, \\
& q_{j}^{\prime}=(b-2) q_{j}\left(\sum_{i=1}^{N} p_{i} e^{-\left|q_{j}-q_{i}\right|}\right)\left(\sum_{i=1}^{N} p_{i} \operatorname{sgn}\left(q_{j}-q_{i}\right) e^{-\left|q_{j}-q_{i}\right|}\right),
\end{aligned}
$$

which leads to the conclusion of Theorem 1.5.

\section{Analysis of the Novikov equation in weighted spaces}

In this section, for the convenience of the readers, we first present some standard definitions. In general, a weight function is simply a non-negative function. A weight function $v: \mathbb{R}^{n} \rightarrow \mathbb{R}$ is called sub-multiplicative if

$$
v(x+y) \leq v(x) v(y) \quad \text { for all } x, y \in \mathbb{R}^{n} .
$$

Given a sub-multiplicative function $v$, a positive function $\phi$ is $v$-moderate if and only if

$$
\exists C_{0}>0: \phi(x+y) \leq C_{0} v(x) \phi(y) \quad \text { for all } x, y \in \mathbb{R}^{n} .
$$


If $\phi$ is $v$-moderate for some sub-multiplicative function $v$, then we say that $\phi$ is moderate. This is the usual terminology in time-frequency analysis papers [34]. Let us recall the most standard examples of such weights. Let

$$
\phi(x)=\phi_{a, b, c, d}(x)=e^{a|x|^{b}}(1+|x|)^{c} \log (e+|x|)^{d} .
$$

We have (see [33]) the following conditions:

(i) For $a, c, d \geq 0$ and $0 \leq b \leq 1$, such weight is sub-multiplicative.

(ii) If $a, c, d \in \mathbb{R}$ and $0 \leq b \leq 1$, then $\phi$ is moderate. More precisely, $\phi_{a, b, c, d}$ is $\phi_{\alpha, \beta, \gamma, \delta}$-moderate for $|a| \leq \alpha,|b| \leq \beta,|c| \leq \gamma$ and $|d| \leq \delta$.

The elementary properties of sub-multiplicative and moderate weights can be found in [33]. Next, we prove Theorem 1.6.

Proof of Theorem 1.6 We define

$$
F(u)=\frac{b-2}{2}\left(\partial_{x} u\right)^{3}+\partial_{x}\left(\frac{b}{3} u^{3}+\frac{6-b}{2} u\left(\partial_{x} u\right)^{2}\right) .
$$

We also introduce the kernel $G(x)=\frac{1}{2} e^{-|x|}$. Then Eq. (1.1) can be rewritten as

$$
u_{t}+u^{2} \partial_{x} u+G * F(u)=0
$$

Note that from the assumption $u \in C\left([0, T], H^{s}\right), s>3 / 2$, we get

$$
M \equiv \sup _{t \in[0, T]}\left(\|u(t)\|_{\infty}+\left\|\partial_{x} u(t)\right\|_{\infty}\right)<\infty
$$

For any $N \in \mathbb{Z}^{+}$, let us consider the $N$-truncations

$$
f(x)=f_{N}(x)= \begin{cases}\phi(x) & \text { if } \phi(x) \leq N \\ N & \text { if } \phi(x)>N\end{cases}
$$

Observe that $f: \mathbb{R} \rightarrow \mathbb{R}$ is a locally absolutely continuous function such that

$$
\|f\|_{\infty} \leq N, \quad\left|f^{\prime}(x)\right| \leq A|f(x)| \quad \text { a.e. }
$$

In addition, if $C_{1}=\max \left\{C_{0}, \alpha^{-1}\right\}$, where $\alpha=\inf _{x \in \mathbb{R}} v(x)>0$, then

$$
f(x+y) \leq C_{1} v(x) f(y), \quad \forall x, y \in \mathbb{R} .
$$

Indeed, let us introduce the set $U_{N}=\{x: \phi(x) \leq N\}$, if $y \in U_{N}$, then $f(x+y) \leq \phi(x+y) \leq$ $C_{0} v(x) f(y)$; if $y \notin U_{N}$, then $f(x+y) \leq N=f(y) \leq \alpha^{-1} v(x) f(y)$.

The constant $C^{1}$ being independent on $N$ shows that the $N$-truncations of a $v$-moderate weight are uniformly $v$-moderate with respect to $N$.

We start considering the case $2 \leq p<\infty$. Multiplying Eq. (4.1) by $f$ and then by $|u f|^{p-2}(u f)$, we get, after integration,

$$
\int_{\mathbb{R}}|u f|^{p-2}(u f)\left(\partial_{t} u f\right) d x+\int_{\mathbb{R}}|u f|^{p}\left(u \partial_{x} u\right) d x+\int_{\mathbb{R}}|u f|^{p-2}(u f)(f \cdot G * F(u)) d x=0 .
$$


Note that the estimates

$$
\int_{\mathbb{R}}|u f|^{p-2}(u f)\left(\partial_{t} u f\right) d x=\frac{1}{p} \frac{d}{d t}\|u f\|_{L^{p}}^{p}=\|u f\|_{L^{p}}^{p-1} \frac{d}{d t}\|u f\|_{L^{p}}
$$

and

$$
\left|\int_{\mathbb{R}}(u f)^{p}\left(u \partial_{x} u\right) d x\right| \leq\left\|u \partial_{x} u\right\|_{L^{\infty}}\|u f\|_{L^{p}}^{p} \leq M^{2}\|u f\|_{L^{p}}^{p}
$$

are true. Moreover, we get

$$
\begin{aligned}
& \left.\left|\int_{\mathbb{R}}\right| u f\right|^{p-2}(u f)[f \cdot(G * F(u))] d x \mid \\
& \leq\|u f\|_{L^{p}}^{p-1}\left\|f \cdot\left\{G *\left[\partial_{x}\left(\frac{b}{3} u^{3}+\frac{6-b}{2} u\left(\partial_{x} u\right)^{2}\right)+\frac{b-2}{2}\left(\partial_{x} u\right)^{3}\right]\right\}\right\|_{L^{p}} \\
& \leq\|u f\|_{L^{p}}^{p-1}\left\{\left\|\left(\partial_{x} G\right) v\right\|_{L^{1}}\left\|f\left(\frac{|b|}{3} u^{3}+\frac{|6-b|}{2} u\left(\partial_{x} u\right)^{2}\right)\right\|_{L^{p}}\right. \\
& \left.\quad+\frac{|b-2|}{2}\|G v\|_{L^{1}}\left\|f\left(\partial_{x} u\right)^{3}\right\|_{L^{p}}\right\} \\
& \leq C M^{2}\|u f\|_{L^{p}}^{p-1}\left(\|u f\|_{L^{p}}+\left\|\left(\partial_{x} u\right) f\right\|_{L^{p}}\right) .
\end{aligned}
$$

In the first inequality we used Hölder's inequality, and in the second inequality we applied Proposition 3.2 in [33], and in the last one we used condition (1.6). Here, $C$ depends only on $v$ and $\phi$. From (4.2) we can obtain

$$
\frac{d}{d t}\|u f\|_{L^{p}} \leq C_{1} M^{2}\|u f\|_{L^{p}}+C_{2} M^{2}\left\|\left(\partial_{x} u\right) f\right\|_{L^{p}} .
$$

Next, we give estimates on $u_{x} f$. Differentiating (4.1) with respect to $x$-variable, next multiplying by $f$ produces the equation

$$
\partial_{t}\left[\left(\partial_{x} u\right) f\right]+u^{2} f \partial_{x}^{2} u+\left[\left(\partial_{x} u\right) f\right]\left(u \partial_{x} u\right)+f\left[\partial_{x} G * F(u)\right]=0 .
$$

Multiplying this equation by $\left|f \partial_{x} u\right|^{p-2}\left(f \partial_{x} u\right)$ with $p \in \mathbb{Z}^{+}$, integrating the result in the $x$-variable, we note that

$$
\int_{\mathbb{R}}\left|f \partial_{x} u\right|^{p-2}\left(f \partial_{x} u\right) \partial_{t}\left[\left(\partial_{x} u\right) f\right] d x=\left\|f \partial_{x} u\right\|_{L^{p}}^{p-1} \frac{d}{d t}\left\|f \partial_{x} u\right\|_{L^{p}}
$$

and

$$
\begin{aligned}
& \left.\left|\int_{\mathbb{R}}\right| f \partial_{x} u\right|^{p-2}\left(f \partial_{x} u\right)\left[f \partial_{x}(G * F(u))\right] d x \mid \\
& \quad \leq\left\|f \partial_{x} u\right\|_{L^{p}}^{p-1}\left\|f \partial_{x}(G * F(u))\right\|_{L^{p}} \\
& \quad \leq C M^{2}\left\|f \partial_{x} u\right\|_{L^{p}}^{p-1}\left(\|u f\|_{L^{p}}+\left\|\left(\partial_{x} u\right) f\right\|_{L^{p}}\right) .
\end{aligned}
$$


In the third inequality we applied the pointwise bound $\left|\partial_{x} G(x)\right| \leq \frac{1}{2} e^{-|x|}$ and the condition

$$
\begin{aligned}
& \left.\left|\int_{\mathbb{R}}\right| f \partial_{x} u\right|^{p-2}\left(f \partial_{x} u\right) u^{2} f \partial_{x}^{2} u d x \mid \\
& \quad=\left.\left|\int_{\mathbb{R}}\right| f \partial_{x} u\right|^{p-2}\left(f \partial_{x} u\right) u^{2}\left[\partial_{x}\left(f \partial_{x} u\right)-\left(\partial_{x} u\right)\left(\partial_{x} f\right)\right] d x \mid \\
& \quad=\left.\left|\int_{\mathbb{R}} u^{2} \partial_{x}\left(\frac{\left|f \partial_{x} u\right|^{p}}{p}\right)-\int_{\mathbb{R}}\right| f \partial_{x} u\right|^{p-2}\left(f \partial_{x} u\right) u^{2}\left(\partial_{x} u\right)\left(\partial_{x} f\right) d x \mid \\
& \quad \leq 2 / p M^{2}\left\|f \partial_{x} u\right\|_{L^{p}}^{p}+A M^{2} \mid f \partial_{x} u \|_{L^{p}}^{p} .
\end{aligned}
$$

In the last inequality we used $\left|\partial_{x} f(x)\right| \leq A f(x)$ for a.e. $x$. Thus, we get

$$
\frac{d}{d t}\left\|f \partial_{x} u\right\|_{L^{p}} \leq C_{3} M^{2}\|u f\|_{L^{p}}+C_{4} M^{2}\left\|\left(\partial_{x} u\right) f\right\|_{L^{p}} .
$$

Now, combing inequalities (4.3) and (4.4) and then integrating yields

$$
\|u(t) f\|_{L^{p}}+\left\|\left(\partial_{x} u\right)(t) f\right\|_{L^{p}} \leq\left(\left\|u_{0} f\right\|_{L^{p}}+\left\|\partial_{x} u_{0} f\right\|_{L^{p}} \exp \left(C M^{2} t\right)\right) \quad \text { for all } t \in[0, T] .
$$

Since $f(x)=f_{N}(x) \uparrow \phi(x)$ as $N \rightarrow \infty$ for a.e. $x \in \mathbb{R}$. Recalling that $u_{0} \phi \in L^{p}(\mathbb{R})$ and $\partial_{x} u_{0} \phi \in$ $L^{p}(\mathbb{R})$, we get

$$
\|u(t) \phi\|_{L^{p}}+\left\|\left(\partial_{x} u\right)(t) \phi\right\|_{L^{p}} \leq\left(\left\|u_{0} \phi\right\|_{L^{p}}+\left\|\partial_{x} u_{0} \phi\right\|_{L^{p}} \exp \left(C M^{2} t\right)\right) \quad \text { for all } t \in[0, T] .
$$

At last, we treat the case $p=\infty$. We have $u_{0}, \partial_{x} u_{0} \in L^{2} \cap L^{\infty}$ and $f(x)=f_{N}(x) \in L^{\infty}$. Hence, we have

$$
\|u(t) f\|_{L^{q}}+\left\|\left(\partial_{x} u\right)(t) f\right\|_{L^{q}} \leq\left(\left\|u_{0} f\right\|_{L^{q}}+\left\|\partial_{x} u_{0} f\right\|_{L^{q}}\right) \exp \left(C M^{2} t\right), \quad q \in[2, \infty) .
$$

The last factor on the right-hand side is independent of $q$. Since $\|f\|_{L^{p}} \rightarrow\|f\|_{L^{\infty}}$ as $p \rightarrow \infty$ for any $f \in L^{\infty} \cap L^{2}$ implies that

$$
\|u(t) f\|_{L^{\infty}}+\left\|\left(\partial_{x} u\right)(t) f\right\|_{L^{\infty}} \leq\left(\left\|u_{0} f\right\|_{L^{\infty}}+\left\|\partial_{x} u_{0} f\right\|_{L^{\infty}}\right) \exp \left(C M^{2} t\right) .
$$

The last factor on the right-hand side is independent of $N$. Now taking $N \rightarrow \infty$ implies that estimate (4.5) remains valid for $p=\infty$.

Proof of Theorem 1.7 We start observing that $\phi^{1 / 3}$ is a $v^{1 / 3}$-moderate weight such that $\left(\phi^{1 / 3}\right)^{\prime}(x) \leq \frac{A}{3} \phi^{1 / 3}(x)$. Moreover, $\inf _{\mathbb{R}} v^{1 / 3}>0$. By condition (1.7), $v^{1 / 3} e^{-|x| / 3} \in L^{3 p}(\mathbb{R})$, hence Hölder's inequality implies that $v^{1 / 3} e^{-|x|} \in L^{1}(\mathbb{R})$. Then Theorem 1.6 applied with $p=3$ to the weight $\phi^{1 / 3}$ yields

$$
\left\|u(t) \phi^{1 / 3}\right\|_{L^{3}}+\left\|\left(\partial_{x} u\right)(t) \phi^{1 / 3}\right\|_{L^{3}} \leq\left(\left\|u_{0} \phi^{1 / 3}\right\|_{L^{3}}+\left\|\partial_{x} u_{0} \phi^{1 / 3}\right\|_{L^{3}} \exp \left(C M^{2} t\right)\right) .
$$

Arguing as in the proof of Theorem 1.6, we get

$$
\frac{d}{d t}\|u f\|_{L^{p}} \leq M^{2}\|u f\|_{L^{p}}+\|f(G * F(u))\|_{L^{p}} \quad \text { for } p<\infty,
$$

where $f(x)=f_{N}(x)=\min \{\phi(x), N\}$. 
On the other hand,

$$
\frac{d}{d t}\left\|f \partial_{x} u\right\|_{L^{p}} \leq C M^{2}\left\|\left(\partial_{x} u\right) f\right\|_{L^{p}}+\left\|f\left(\partial_{x} G * F(u)\right)\right\|_{L^{p}} \quad \text { for } p<\infty .
$$

Note that $F(u)=\frac{b-2}{2}\left(\partial_{x} u\right)^{3}+\partial_{x}\left(\frac{b}{3} u^{3}+\frac{6-b}{2} u\left(\partial_{x} u\right)^{2}\right)$ and

$$
\begin{aligned}
& \|f(G * F(u))\|_{L^{p}} \\
& \quad \leq C\left(\left\|\left(\partial_{x} G\right) v\right\|_{L^{p}}\left\|\phi\left(\frac{b}{3} u^{3}+\frac{6-b}{2} u\left(\partial_{x} u\right)^{2}\right)\right\|_{L^{1}}+\frac{|b-2|}{2}\|G v\|_{L^{p}}\left\|\phi\left(\partial_{x} u\right)^{3}\right\|_{L^{1}}\right) \\
& \quad \leq C\left(\left\|\phi^{1 / 3} u\right\|_{L^{3}}^{3}+\left\|\phi u\left(\partial_{x} u\right)^{2}\right\|_{L^{1}}+\left\|\phi^{1 / 3}\left(\partial_{x} u\right)\right\|_{L^{3}}^{3}\right) \\
& \quad \leq C\left(\left\|\phi^{1 / 3} u\right\|_{L^{3}}^{3}+\left\|\phi^{1 / 3} u\right\|_{L^{3}}\left\|\phi^{2 / 3}\left(\partial_{x} u\right)^{2}\right\|_{L^{3 / 2}}+\left\|\phi^{1 / 3}\left(\partial_{x} u\right)\right\|_{L^{3}}^{3}\right) \\
& \quad \leq K_{0} \exp \left(3 C M^{2} t\right) .
\end{aligned}
$$

The constant on the right-hand side is dependent on $N$. Similarly, recalling that $\partial_{x}^{2} G=$ $G-\delta$, we obtain

$$
\begin{aligned}
\left\|f\left(\partial_{x} G * F(u)\right)\right\|_{L^{p}} \leq & \left\|f\left(G *\left(u^{3}+\frac{3}{2} u\left(\partial_{x} u\right)^{2}\right)\right)\right\|_{L^{p}}+\left\|f\left(u^{3}+\frac{3}{2} u\left(\partial_{x} u\right)^{2}\right)\right\|_{L^{p}} \\
& +\left\|f\left(\partial_{x} G *\left(\partial_{x} u\right)^{3}\right)\right\|_{L^{p}} \\
\leq & K_{0} \exp \left(3 C M^{2} t\right)+C M^{2}\left(\|u f\|_{L^{p}}+\left\|\left(\partial_{x} u\right) f\right\|_{L^{p}}\right) .
\end{aligned}
$$

Plugging the two last estimates in (4.6)-(4.7) and summing, we obtain

$$
\frac{d}{d t}\left(\|u(t) f\|_{L^{p}}+\left\|\left(\partial_{x} u\right)(t) f\right\|_{L^{p}}\right) \leq K_{1} M^{2}\left(\left\|u_{0} f\right\|_{L^{p}}+\left\|\partial_{x} u_{0} f\right\|_{L^{p}}\right)+2 K_{0} \exp \left(3 C M^{2} t\right) .
$$

Integrating and finally letting $N \rightarrow \infty$ yields the conclusion in the case $2 \leq p<\infty$. The constants throughout the proof are independent on $p$. Therefore, for $p=\infty$, one can rely on the result established for finite exponents $q$, and then let $q \rightarrow \infty$. The rest argument is fully similar to that of Theorem 1.6.

\section{Competing interests}

The authors declare that they have no competing interests.

\section{Authors' contributions}

This paper is the result of joint work of all authors who contributed equally to the final version of this paper. All authors read and approved the final manuscript.

\section{Author details}

${ }^{1}$ College of Mathematics Science, Chongqing Normal University, Chongqing, 401331, P.R. China. ${ }^{2}$ College of Continuing Education, Chongqing Normal University, Chongqing, 400047, P.R. China.

\section{Acknowledgements}

The authors are very grateful to the anonymous reviewers and editors for their careful read and useful suggestions, which greatly improved the presentation of the paper. This work is supported in part by NSFC grant 11301573 and in part by the Program of Chongqing Innovation Team Project in University under Grant No. KJTD201308. 


\section{References}

1. Novikov, VS: Generalizations of the Camassa-Holm equation. J. Phys. A 42, 342002 (2009)

2. Mikhailov, AV, Novikov, VS: Perturbative symmetry approach. J. Phys. A 35, 4775-4790 (2002)

3. Hone, ANW, Wang, JP: Integrable peakon equations with cubic nonlinearity. J. Phys. A 41, 372002 (2008)

4. Hone, ANW, Lundmark, H, Szmigielski, J: Explicit multipeakon solutions of Novikov's cubically nonlinear integrable Camassa-Holm equation. Dyn. Partial Differ. Equ. 6, 253-289 (2009)

5. $\mathrm{Wu}, \mathrm{XL}$, Yin, ZY: A note on the Cauchy problem of the Novikov equation. Appl. Anal. (2013). doi:10.1080/00036811.2011.649735

6. Yan, W, Li, YS, Zhang, YM: The Cauchy problem for the integrable Novikov equation. J. Differ. Equ. 253, 298-318 (2012)

7. Ni, LD, Zhou, Y: Well-posedness and persistence properties for the Novikov equation. J. Differ. Equ. 250, 3002-3201 (2011)

8. Himonas, A, Holliman, C: The Cauchy problem for the Novikov equation. Nonlinearity 25, 449-479 (2012)

9. Grayshan, K: Peakon solutions of the Novikov equation and properties of the data-to-solution map. J. Math. Anal. Appl. 397, 515-521 (2013)

10. Yan, W, Li, YS, Zhang, YM: The Cauchy problem for the Novikov equation. Nonlinear Differ. Equ. Appl. (2013). doi:10.1007/s00030-012-0202-1

11. Tiglay, F: The periodic Cauchy problem for Novikov's equation. Int. Math. Res. Not. 20, 4633-4648 (2011)

12. Jiang, ZH, Ni, LD: Blow-up phenomena for the integrable Novikov equation. J. Math. Anal. Appl. 385, $551-558$ (2012)

13. $\mathrm{Wu}, \mathrm{SY}$, Yin, ZY: Global weak solutions for the Novikov equation. J. Phys. A 44, 055202 (2011)

14. Lai, SY, Li, N, Wu, YH: The existence of global strong and weak solutions for the Novikov equation. J. Math. Anal. Appl. 399, 682-691 (2013)

15. Dullin, HR, Gottwald, GA, Holm, DD: Camassa-Holm, Korteweg-de Vries-5 and other asymptotically equivalent equations for shallow water waves. Fluid Dyn. Res. 33, 73-79 (2003)

16. Dullin, HR, Gottwald, GA, Holm, DD: On asymptotically equivalent shallow water wave equations. Physica D 190, 1-14 (2004)

17. Dullin, HR, Gottwald, GA, Holm, DD: An integrable shallow water equation with linear and nonlinear dispersion. Phys. Rev. Lett. 87, 4501-4504 (2001)

18. Degasperis, A, Procesi, M: Asymptotic integrability. In: Degasperis, A, Gaeta, G (eds.) Symmetry and Perturbation Theory, pp. 23-37. World Scientific, Singapore (1999)

19. Degasperis, A, Holm, DD, Hone, ANW: Integral and non-integrable equations with peakons. In: Nonlinear Physics: Theory and Experiment, II (Gallipoli, 2002), pp. 37-43. World Scientific, River Edge (2003)

20. Ivanov, Rl: Water waves and integrability. Philos. Trans. R. Soc. Lond. A 365, 2267-2280 (2007)

21. Holm, DD, Staley, MF: Wave structure and nonlinear balances in a family of evolutionary PDEs. SIAM J. Appl. Dyn. Syst. 2, 323-380 (2003)

22. Holm, DD, Staley, MF: Nonlinear balance and exchange of stability in dynamics of solitons, peakons, ramps/cliffs and leftons in a 1-1 nonlinear evolutionary PDE. Phys. Lett. A 308, 437-444 (2003)

23. Escher, J, Yin, Z: Well-posedness, blow-up phenomena, and global solutions for the $b$-equation. J. Reine Angew. Math. 624, 51-80 (2008)

24. Niu, W, Zhang, S: Blow-up phenomena and global existence for the nonuniform weakly dissipative $b$-equation. J. Math. Anal. Appl. 374, 166-177 (2011)

25. Gui, GL, Liu, Y, Tian, TX: Global existence and blow-up phenomena for the peakon b-family of equations. Indiana Univ. Math. J. 57, 1209-1234 (2008)

26. $\mathrm{Mi}, \mathrm{YS}, \mathrm{Mu}, \mathrm{CL}$ : On the Cauchy problem for the modified Novikov equation with peakon solutions. J. Differ. Equ. 254 961-982 (2013)

27. Danchin, R: A note on well-posedness for Camassa-Holm equation. J. Differ. Equ. 192, 429-444 (2003)

28. Danchin, R: A few remarks on the Camassa-Holm equation. Differ. Integral Equ. 14, 953-988 (2001)

29. Himonas, AA, Misiolek, G, Ponce, G, Zhou, Y: Persistence properties and unique continuation of solutions of the Camassa-Holm equation. Commun. Math. Phys. 271, 511-522 (2007)

30. Ni, LD, Zhou, Y: A new asymptotic behavior of solutions to the Camassa-Holm equation. Proc. Am. Math. Soc. 140, 607-614 (2012)

31. Henry, D: Persistence properties for a family of nonlinear partial differential equations. Nonlinear Anal. 70, 1565-1573 (2009)

32. Zhou, Y: On solutions to the Holm-Staley b-family of equations. Nonlinearity 23, 369-381 (2010)

33. Brandolese, L: Breakdown for the Camassa-Holm equation using decay criteria and persistence in weighted spaces. Int. Math. Res. Not. 22, 5161-5181 (2012)

34. Aldroubi, A, Gröchenig, K: Nonuniform sampling and reconstruction in shift-invariant spaces. SIAM Rev. 43, 585-620 (2001)

10.1186/1029-242X-2013-560

Cite this article as: Zhou and Chen: A few remarks on the generalized Novikov equation. Journal of Inequalities and Applications 2013, 2013:560 